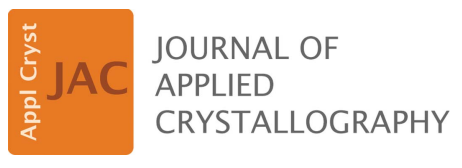

ISSN 1600-5767

Received 1 June 2015

Accepted 19 August 2015

Edited by G. Kostorz, ETH Zurich, Switzerland

Keywords: polarization analysis; neutron spin filters; small-angle neutron scattering; dynamic nuclear polarization.

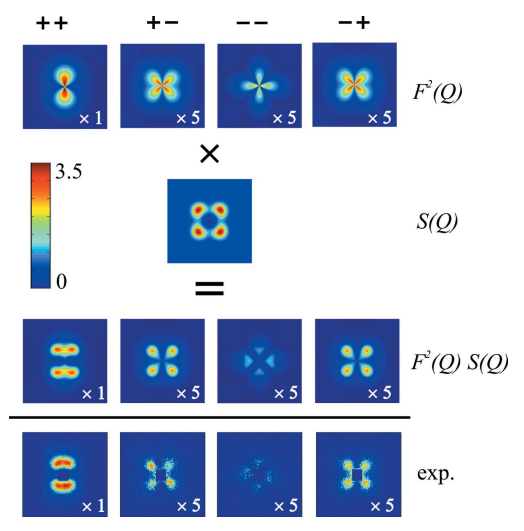

C 2015 International Union of Crystallography

\section{Polarization analysis in neutron small-angle scattering with a novel triplet dynamic nuclear polarization spin filter}

\author{
N. Niketic, ${ }^{a}$ B. van den Brandt, ${ }^{a}$ W. Th. Wenckebach, ${ }^{a}$ J. Kohlbrecher ${ }^{b}$ and P. \\ Hautle $^{\mathrm{a}_{*}}$
}

\begin{abstract}
a Laboratory for Scientific Developments and Novel Materials (LDM), Paul Scherrer Institute, CH-5232 Villigen PSI, Switzerland, and ${ }^{\mathbf{b}}$ Laboratory for Neutron Scattering (LNS), Paul Scherrer Institute, CH-5232 Villigen PSI, Switzerland.

*Correspondence e-mail: patrick.hautle@psi.ch
\end{abstract}

A novel neutron spin filter whose principle is based on the strong spin dependence of the neutron scattering on protons has been developed. The dimensions of this filter are small, and it works very efficiently and is stable even in inhomogeneous fields. The protons in the naphthalene spin filter crystal are polarized by a recently developed method of dynamic nuclear polarization using photoexcited triplet states. This technique allows the design of a very compact apparatus that can be placed at a close distance to the sample under investigation. The application of this filter as a polarization analyzer is demonstrated in a magnetic small-angle neutron scattering experiment with the measurement of the spin-dependent scattering signals of a $\mathrm{CuFeNi}$ alloy. This sample has a pronounced textured structure factor of ferromagnetic precipitates in a paramagnetic matrix. The performance of the spin filter as an analyzer is illustrated by the excellent agreement of the experimental data with simulations based on a model of homogeneously magnetized spherical particles which are ordered in a simple cubic paracrystalline lattice.

\section{Introduction}

Magnetic small-angle neutron scattering (SANS) is presently the only technique allowing resolution of the spin microstructure on the nanometre length scale $(1-200 \mathrm{~nm})$ of precipitates in an nonmagnetic matrix. It measures the diffuse scattering along the forward direction, which arises from nanoscale variations in both the magnitude and the orientation of the magnetization vector field. Using polarized incident neutrons the contributions of the nonmagnetic and magnetic scattering of the same nanostructure can be separated by exploiting the cross term between nuclear and magnetic scattering, which changes its sign with the change of the polarization of the incident neutron beam (Moon et al., 1969). However, this cross term only depends on the component of the magnetic moments aligned parallel to the applied field and incident neutrons with their spin state either parallel or antiparallel to the field and will measure only the non-spin-flip scattering process. Only the separate determination of the socalled spin-flip process obtained by a polarization analysis of the scattered neutrons gives access to the magnetic moment perpendicular to the applied field. This information is crucial for the understanding of the micromagnetic response of magnetic materials in intermediate non-saturating fields (Wiedenmann, 2005; Michels, 2014).

Polarization analysis in SANS experiments became possible only recently with progress in the efficiency of ${ }^{3} \mathrm{He}$ spin filters (Chen et al., 2014), allowing the neutron spins from a diver- 
gently scattered beam to be analyzed in the forward direction. With this rather new option, key outstanding questions in studies with both fundamental and technological relevance could be addressed; examples include the study of interfacial magnetic effects in nanoscopic heterostructures and the manipulation of magnetism with strain and electrical current (Dufour et al., 2011; Fitzsimmons \& Schuller, 2014), electric field-induced magnetization in multiferroics (Ueland et al., 2010; Ramazanoglu et al., 2011), magnetostriction in Fe-Ga alloys (Laver et al., 2010), three-dimensional magnetic morphology in nanoparticles (Krycka et al., 2010), and studies on the intraparticle spin misalignment in arrays of nanostructured materials (Honecker et al., 2010).

One of the main practical problems of integrating a ${ }^{3} \mathrm{He}$ spin filter into the experimental environment is its sensitivity to stray magnetic fields. In order to limit relaxation of the ${ }^{3} \mathrm{He}$ polarization, i.e. the decrease of filter analyzing efficiency, the ${ }^{3} \mathrm{He}$ filter cell has to be placed in a very homogeneous magnetic field environment $\left(\Delta B / B<5 \times 10^{-4} \mathrm{~cm}^{-1}\right)$, which is difficult in a sample environment with magnetic fields. This is typical for the investigation of magnetic scattering. The filter cell needs to be placed in a magnetostatic shielding box at some distance $(\sim 1 \mathrm{~m})$ from the sample under investigation (Petoukhov et al., 2006; Chen et al., 2009). The technical solutions realized achieve a typical angular coverage for SANS of $Q \simeq 0.4 \mathrm{~nm}^{-1}$.

Here we demonstrate the use of an alternative neutron spin filter based on the strong spin dependence of the neutron scattering on protons. It is small and works in inhomogeneous fields. The spin filter is operated at $32 \mathrm{~mm}$ distance from the sample under investigation in a common magnetic field. This results in a compact setup and yields a large acceptance angle that is comparable to ${ }^{3} \mathrm{He}$ spin filter setups, even with a moderate sized analyzer. Furthermore, no guide field is needed to transport the neutron spin adiabatically from the sample to the analyzer. We create the necessary large proton polarization in a single crystal of naphthalene doped with deuterated pentacene using a recently developed method of dynamic nuclear polarization (DNP) (Henstra \& Wenckebach, 2013; Eichhorn, van den Brandt et al., 2013) that uses photoexcited triplet states (Henstra et al., 1990) and requires only moderate experimental means (Eichhorn, Haag et al., 2013). Proton polarization values above $70 \%$ are achieved at a field of $0.36 \mathrm{~T}$ using a simple flow cryostat for cooling (Eichhorn et al., 2014).

We first briefly review the underlying physical principles that characterize the efficiency of a spin filter based on polarized protons. $\$ 3$ gives experimental details of the apparatus and describes its setup and mode of operation in a SANS polarization analysis experiment. The performance of the filter is then illustrated by comparing the experimental data with simulations based on a structural model of the magnetic sample under investigation.

\section{Neutron polarization analysis with polarized protons}

Lushchikov et al. (1969) demonstrated that dynamically polarized proton spins offer an attractive possibility to realize a broad-band neutron spin filter, as the spin-dependent neutron-proton cross section is large in a broad wavelength range. The working principle of a polarized proton spin filter is based on the fact that the singlet cross section for neutronproton scattering is much higher than the triplet cross section (Glättli \& Goldman, 1987). Hence, neutrons polarized antiparallel to the protons are much more strongly scattered than those polarized parallel. This applies for coherent and incoherent scattering. It is customary to write the cross section as

$$
\sigma_{ \pm}=\sigma_{0} \pm \sigma_{\mathrm{p}} P
$$

where the + sign and the - sign stand for the two eigenstates of the neutron spin with respect to the direction of the proton spin polarization. The second term is the product of the 'polarization cross section' $\sigma_{\mathrm{p}}$ and the proton spin polarization $P$. The first term $\sigma_{0}$ is independent of the neutron spin polarization and either independent of $P$ or a symmetric function of $P$.

The transmissions of the two spin components of the unpolarized neutron beam through a spin filter with a proton density $N$ and a thickness $d$ are given by

$$
T_{ \pm}=\frac{1}{2} \exp \left[-\left(\sigma_{0} \pm \sigma_{\mathrm{p}} P N d\right)\right] .
$$

Thus an initially unpolarized neutron beam acquires a polarization

$$
A=\frac{T_{+}-T_{-}}{T_{+}+T_{-}}=\tanh \left(\sigma_{\mathrm{p}} P N d\right),
$$

which is called the filter analyzing power. The thicker the filter, the higher the filter analyzing power for a given $\left(\sigma_{\mathrm{p}} N\right)$ and the lower the transmission

$$
T=T_{+}+T_{-}=\exp \left(-\sigma_{0} N d\right) \cosh \left(\sigma_{\mathrm{p}} P N d\right)
$$

of the neutron beam. Note that for a given thickness $d$ the total transmission $T$ increases with the filter polarization $P$.

Structural effects on the values of $\sigma_{\mathrm{p}}$ and $\sigma_{0}$ observed for slow neutrons are difficult to predict theoretically, even when the crystal structure is well known. So, the wavelength dependencies of these cross sections need to be determined experimentally for each material. Fig. 1 of Eichhorn et al. (2014) gives the experimental values for naphthalene. In the wavelength range relevant for SANS, i.e. above $5 \AA$, neutrons are scattered incoherently out of the beam and $\sigma_{\mathrm{p}}(\lambda)$ is independent of the host crystal of the proton spins. The spin incoherent part of the cross section per nucleus is given by (Williams, 1988)

$$
\sigma_{\text {inc }}=\pi b_{\mathrm{N}}^{2}\left[I(I+1)-I^{2} P^{2}-I p P\right],
$$

where $b_{\mathrm{N}}=-(5.824 \pm 0.002) \times 10^{-12} \mathrm{~cm}$ is the spin-dependent scattering length difference (Dianoux \& Lander, 2003), $I=\frac{1}{2}$ and $p$ is the neutron spin polarization. We also have to take into account absorption, which occurs mainly through the singlet channel and is thus polarization dependent. One can show that the polarization-dependent capture cross section $\sigma_{\mathrm{p}}^{\mathrm{c}}=-\sigma_{0}^{\mathrm{c}} \quad$ (Glättli \& Goldman, 1987), where $\sigma_{0}^{\mathrm{c}}=$ $(0.1849 \pm 0.0004) \times 10^{-24} \mathrm{~cm}^{2} \AA^{-1}$ is the polarization-independent capture cross section (Dianoux \& Lander, 2003). We 


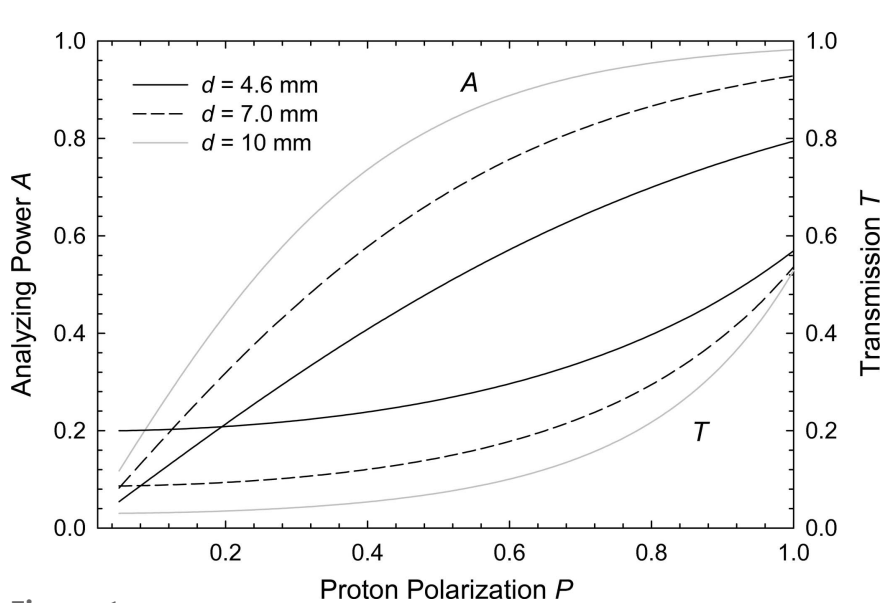

Figure 1

Analyzing power (left axis) and transmission (right axis) for different thicknesses $d$ of the naphthalene filter with a proton density $N=4.330 \times 10^{22} \mathrm{~cm}^{-3}$ for a cold neutron beam with a wavelength of $\lambda=6 \AA$. The solid line gives the values for the $4.6 \mathrm{~mm}$-thick sample used in the SANS experiment.

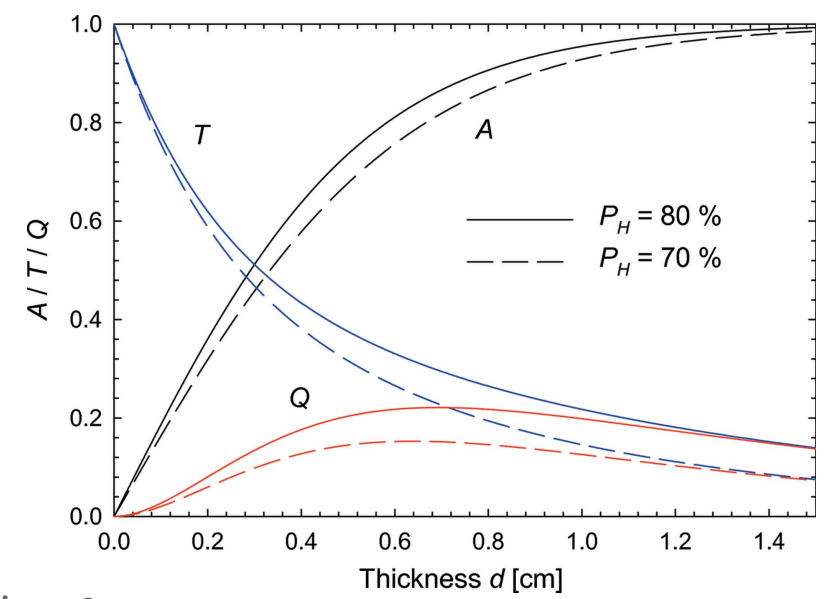

Figure 2

Performance of a naphthalene neutron spin filter with a proton spin polarization of $P=0.7$ (dashed lines) and $P=0.8$ (solid lines) for a cold neutron beam with a wavelength of $\lambda=6 \AA$. Plotted are the analyzing (polarizing) efficiency $(A)$, total transmission $(T)$ and figure of merit $Q$ as a function of the filter thicknesses $d$.

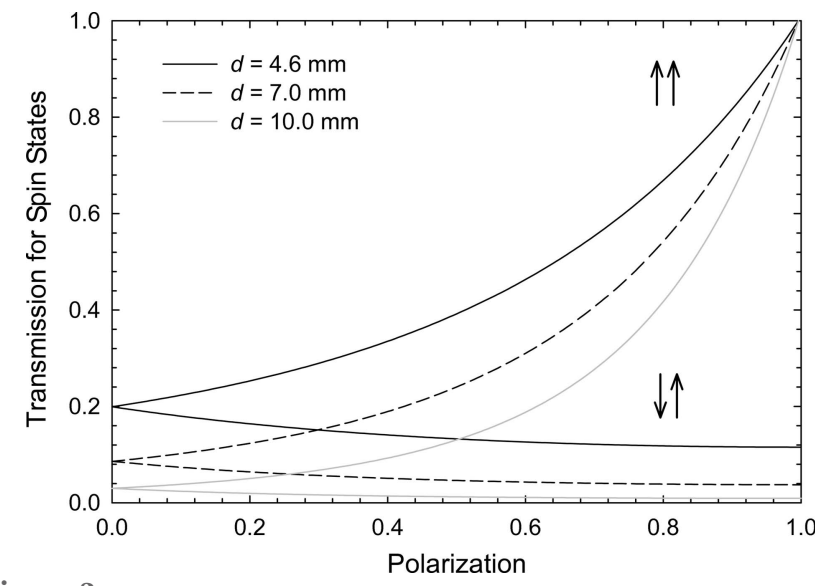

Figure 3

Transmissions of the two neutron spin orientations of a beam of $6 \AA$ wavelength through a naphthalene filter of $4.6,7$ and $10 \mathrm{~mm}$ thickness, as function of the proton polarization. Note, their sum divided by 2 gives the transmission of an unpolarized beam plotted in Fig. 1. obtain for the polarization cross section in the range relevant for SANS

$$
\sigma_{\mathrm{p}}(\lambda) \simeq-[53.3+0.185 \lambda(\AA)] \times 10^{-24} \mathrm{~cm}^{2} .
$$

Likewise we obtain for the cross section independent of the neutron polarization

$$
\sigma_{0}(\lambda) \simeq\left[79.9\left(1-\frac{1}{3} P^{2}\right)+0.185 \lambda(\AA)\right] \times 10^{-24} \mathrm{~cm}^{2} .
$$

Inserting these cross sections into equations (3) and (4), we can estimate the performance of the naphthalene filter with a proton density $N=4.330 \times 10^{22} \mathrm{~cm}^{-3}$ as a function of the polarization for different thicknesses. As is illustrated in Fig. 1, a proton polarization of $P \simeq 0.7$ produces a high analyzing power with a considerable total transmission. Often the socalled figure of merit $Q=A^{2} T$ is taken as the statistically relevant factor to optimize the filter performance (Williams, 1988). This quality factor derived assuming a pure spin-flip signal also applies to the experimental case studied in the following, where the strength of the magnetic scattering is comparable to the nuclear scattering. For polarization values between 0.7 and 0.8 a filter thickness of $d \simeq 7 \mathrm{~mm}$ is ideal for neutrons in the wavelength range we consider here (Fig. 2). However, if a very weak spin-flip signal needs to be separated from a commensurate non-spin-flip signal, it is preferable to have a thicker filter than suggested by the figure of merit. A detailed discussion for the case of ${ }^{3} \mathrm{He}$ spin filters is given by Goossens \& Cussen (1999) and Gentile et al. (2005), and the statistical arguments therein apply to proton spin filters as well.

In the case of use as a polarization analyzer, the transmission of the neutron spin components both parallel and antiparallel to the proton spin polarization are relevant and are given for three filter thicknesses (see Fig. 3).

\section{Experimental methods}

\subsection{Triplet spin filter}

Traditionally, methods of DNP (Abragam \& Goldman, 1978) have been used to polarize protons in solid samples, and spin polarizations near unity have been achieved employing the classical DNP scheme: electron spins are polarized by cooling them to a low temperature $(\sim 1 \mathrm{~K})$ in a strong magnetic field $(2.5-5 \mathrm{~T})$ and their high polarization is transferred to the nuclear spins by means of a microwave field. For some applications, such as polarization analysis in SANS, the necessary cryogenics and magnets are prohibitive. This drawback can be circumvented with the more recent method of 'triplet DNP', which uses the electron spin of photoexcited triplet states (Henstra et al., 1990) that becomes polarized as a result of the selection rules of the optical excitation process. In our case of a naphthalene crystal doped with a small concentration of pentacene- $d_{14}$ guest molecules, the 'effective' electron spin polarization of the pentacene triplet state is $P_{\mathrm{e}} \simeq 0.9$ (Van Strien \& Schmidt, 1980) at a field above $0.1 \mathrm{~T}$. This high electron polarization is most efficiently transferred to the nuclear spins by means of the integrated solid effect 
(ISE) (Henstra \& Wenckebach, 2013; Eichhorn, van den Brandt et al., 2013). In this method the externally applied magnetic field is adiabatically swept over the inhomogeneously broadened electron spin resonance (ESR) line (typically in $10 \mu \mathrm{s})$ under microwave irradiation, which allows all electron spins to contribute constructively to the polarization transfer. Since the proton spin-lattice relaxation in naphthalene is very long already at temperatures below $100 \mathrm{~K}$, the cycle can be repeated many times, and the proton spin polarization is accumulated and can approach the electron spin polarization.

The filter crystal is cut out of a large single crystal grown with a self-seeding vertical Bridgman technique from zonerefined naphthalene doped with pentacene- $d_{14}$. Its size is $4.2 \times 5 \mathrm{~mm}$ with a thickness of $d=4.6 \mathrm{~mm},{ }^{1}$ where its bottom/top corresponds to the crystal ac plane. The pentacene concentration was determined with optical transmission spectroscopy to be $(3 \pm 0.5) \times 10^{-5} \mathrm{~mol} / \mathrm{mol}$. It is mounted on a polychlorotrifluorethylene holder, introduced into a helium flow cryostat, and cooled to temperatures between 25 and $100 \mathrm{~K}$. The pentacene molecules are excited into the triplet state with a disc laser system operating at $515 \mathrm{~nm}$, generating pulses of $400 \mathrm{~ns}$ at a repetition rate set to $1 \mathrm{kHz}$. The light is transported from the laser system conveniently located about $10 \mathrm{~m}$ away via a multimode fiber to an optical stage at the bottom of the cryostat, collimating the unpolarized light to a beam waist of about $9 \mathrm{~mm}$. The beam axis is vertical and along the $b$ axis, illuminating the sample crystal homogeneously. The energy per pulse at the entrance window of the cryostat is approximately $1 \mathrm{~mJ}$.

A small electromagnet provides the static magnetic field and DNP is performed on the so-called high-field transition $\left(S_{z}=0 \leftrightarrow 1\right)$ at $B \simeq 0.36 \mathrm{~T}$ using a pulsed ESR system operating at $9.3 \mathrm{GHz}$ and synchronized to the laser. The latter also enables us to observe the ESR signal of the triplet states and orient the static magnetic field along the $X$ axis of the pentacene molecules. A pulsed NMR system is used to monitor the proton polarization and to optimize the parameters for the ISE process. A detailed description of the triplet DNP apparatus is given by Eichhorn, Haag et al. (2013).

\subsection{Experimental arrangement}

The neutron experiments were performed at the SANS I instrument (Aswal et al., 2008) at the continuous spallation neutron source SINQ at the Paul Scherrer Institute in Switzerland. A layout of the experimental setup is given in Fig. 4. The incoming beam was monochromated by a velocity selector to a wavelength of $\lambda=0.6 \mathrm{~nm}$ with a spread of $\Delta \lambda / \lambda=10 \%$ and polarized with a V-shaped $\mathrm{Fe} / \mathrm{Si}$ supermirror transmission polarizer to 0.98 at the collimations used. The neutron polarization could be reversed by means of an adiabatic spin flipper with an efficiency of 0.99 .

The sample, a small platelet of CuNiFe alloy (see §4.1), was placed on the outside of the flow cryostatat $32 \mathrm{~mm}$ in front of

\footnotetext{
$\mathbf{1}$ The filter size and thickness are limited by the presently employed microwave cavity. For a further discussion see $§ 5$.
}

the analyzer, i.e. it was at room temperature but still in the homogeneous magnetic field of the electromagnet pointing perpendicular to the neutron beam. Inside the cryostat a $3 \times 3 \mathrm{~mm}$ cadmium aperture placed at a distance of $14 \mathrm{~mm}$ from the polarized naphthalene crystal defined the analyzer cross section. The close sample-to-analyzer distance was ample to cover the necessary $Q$ range of the scattering, even with the relatively small analyzer cross section. At a wavelength of $0.6 \mathrm{~nm}$ the accessible $Q$ range was $0.41 \mathrm{~nm}^{-1}$.

\subsection{Mode of operation}

To perform a proper polarization analysis of the SANS experiment with all necessary corrections for the imperfections of analyzer, polarizer and spin flipper, four partial neutron intensities, $I_{\exp }^{++}, I_{\exp }^{-+}, I_{\exp }^{+-}, I_{\exp }^{--}$, need to be measured together with the corresponding analyzer transmissions (Wildes, 2006; Honecker et al., 2010; Krycka, Chen et al., 2012). To do so, first the spin filter is polarized to a sufficiently high level of $P>0.7$. After switching off the laser excitation light the polarization stays practically frozen and the magnetic field can be set to the value desired for the SANS measurement. Now two partial scattering intensities are measured, e.g. $I_{\text {exp }}^{++}$ and $I_{\text {exp }}^{-+}$with the analyzer polarized positive and neutron spin polarized positive and negative with respect to the magnetic field applied to the sample. Then the analyzer is polarized to the other direction and the remaining two neutron intensities $I_{\exp }^{+-}$and $I_{\exp }^{--}$are measured. The analyzer polarization is monitored regularly by short transmission measurements.

In the present experiment the polarization of the spin filter was built up with a time constant of $\tau \simeq 200 \mathrm{~min}$ to a level $P>0.7$. The magnetic field was set at values between 520 and $72 \mathrm{mT}$. Operating the flow cryostat at $25 \mathrm{~K}$, the relaxation times $T_{1}$ of the analyzer polarization were very long $(320 \mathrm{~h}$ at $520 \mathrm{mT}, 133 \mathrm{~h}$ at $72 \mathrm{mT}$ ) and had a negligible influence on the filter efficiency on the timescale of a single neutron measurement, which typically took $15 \mathrm{~min}$. The transmissions for the two neutron spin states through the spin filter apparatus with the analyzer crystal polarized to $70 \%$ were $T_{\exp }^{++}=0.51(4)$ and $T_{\exp }^{-+}=0.11(0)$, respectively, compared to the empty beam. The transmission of the apparatus itself without analyzer crystal was measured to be $91 \%$. Including the corrections for the non-ideal beam polarization, this

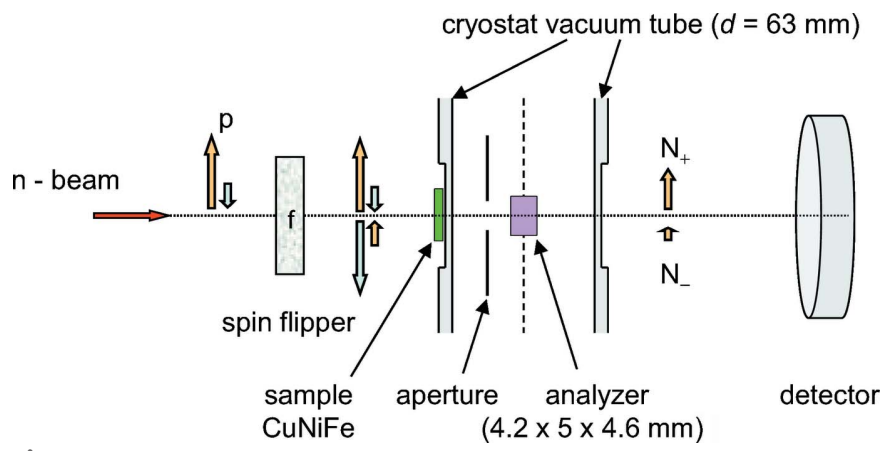

Figure 4

Scheme of the experimental setup with the arrangement of the filter, sample and cryostat (not to scale). 
amounts to a transmission of $T^{++}=0.57(2)$ and $T^{-+}=0.11$ (3) for the filter crystal itself, which is in good agreement with the theoretical expectation (see Fig. 3).

\section{Experiment and results}

To test the performance of the spin filter we chose a sample that exhibits a strong spin-dependent scattering pattern resulting from comparable nuclear and magnetic contrasts. It was a small platelet of a $\mathrm{CuNiFe}$ alloy where the nanometre sized ferromagnetic $\mathrm{NiFe}$-rich clusters are embedded in the paramagnetic crystalline copper-rich matrix (Wagner \& Kohlbrecher, 2005). CuNiFe alloys have been extensively studied in the past by means of transmission electron microscopy (Wahi \& Stajer, 1984), analytical field ion microscopy (FIM) (Piller et al., 1984) and scattering techniques, mostly SANS (Wagner, 1988; Wagner \& Kohlbrecher, 2005) and small-angle X-ray scattering (Lyon \& Simons, 1992, 1994). Our aim was to asses the potential of our spin filter apparatus by comparing the experimental SANS data with simulations modeling the precipitate morphology of our sample.

\subsection{Sample material}

The alloy with the nominal composition $\mathrm{Cu}_{68 \text { at. } \%} \mathrm{Ni}_{24 a t . \%}{ }^{-}$ $\mathrm{Fe}_{8 \text { at.\% }}$ was prepared by levitation melting in an induction furnace. The ingot was $50 \%$ cold-worked by pressing, homogenized by solution annealing at $1300 \mathrm{~K}$ for $2 \mathrm{~h}$ and water quenched. The pressed ingot of about $3 \mathrm{~mm}$ in thickness was then cold rolled to foils of $1 \mathrm{~mm}$ thickness by five consecutive rolling passes and cut into pieces of $2 \mathrm{~cm}^{2}$. The rolling of the sample is known to introduce a very pronounced texture. For face-centered cubic metals (Delannay et al., 2001) a pronounced $\{100\}\langle 110\rangle$ rolling texture is known. After rolling, the sample was solution annealed at $1073 \mathrm{~K}$ for $2 \mathrm{~h}$, followed by a water quench. The final annealing was then done at $823 \mathrm{~K}$ for $967 \mathrm{~h}$. At this temperature the previously homogeneous system decomposes into a $\mathrm{Cu}$-rich $\alpha$ phase and an $\mathrm{Ni} / \mathrm{Fe}$-rich $\alpha^{\prime}$ phase. The equilibrium volume fraction of the $\alpha^{\prime}$ phase is about $22 \%$. From FIM experiments the composition is known both for the ferromagnetic phase $\left(\mathrm{Cu}_{6.7 \mathrm{at} . \%} \mathrm{Ni}_{58 \mathrm{at} . \%} \mathrm{Fe}_{34.8 \mathrm{at} . \%}\right)$ and for the paramagnetic matrix $\left(\mathrm{Cu}_{85.5 \text { at.\% }} \mathrm{Ni}_{13.6 a t . \%} \mathrm{Fe}_{0.9 \mathrm{at} . \%}\right)$ (Wagner \& Kohlbrecher, 2005). From the composition of the precipitates and the matrix one can estimate that the magnetic scattering contrast should be about 2.4 times as large as the nuclear scattering contrast. The calculated values are listed in Table 1. The magnetic moments per atom of pure iron and pure nickel are $2.216 \mu_{\mathrm{B}}$ and $0.616 \mu_{\mathrm{B}}$, and the magnetizations at $0 \mathrm{~K}$ are $1.75 \times 10^{6} \mathrm{~A} \mathrm{~m}^{-1}$ and $0.51 \times 10^{6} \mathrm{~A} \mathrm{~m}^{-1}$, respectively. The saturation magnetization is reduced at room temperature by $2 \%$ for $\mathrm{Fe}$ and $6 \%$ for $\mathrm{Ni}$ according to Crangle \& Goodman (1971). As no bulk magnetization values were available for the exact composition of the precipitates, the magnetic contrast was extrapolated from the values of pure iron and nickel assuming simple linear mixing. It was further assumed that the matrix is paramagnetic and does not contribute to the magnetic contrast.
Table 1

The magnetic properties of $\mathrm{Fe}$ and $\mathrm{Ni}$ are taken from Crangle \& Goodman (1971) and are used to estimate the scattering length densities of the ferromagnetic precipitates $\mathrm{Cu}_{6.7 \mathrm{at} . \%} \mathrm{Ni}_{58 \mathrm{at} . \%} \mathrm{Fe}_{34.8 \mathrm{at} . \%}$ and the paramagnetic matrix $\mathrm{Cu}_{85.5 \text { at. } \%} \mathrm{Ni}_{13.6 \text { at. } \%} \mathrm{Fe}_{0.9 \text { at.\%. }}$.

The calculated scattering length density difference $\Delta \eta$ between precipitates and matrix is given for nuclear and magnetic scattering, where the latter is compared with our experimental value given in the last row. $\rho$ is the mass density and $V_{\text {at }}$ is the atomic volume.

\begin{tabular}{lllllll}
\hline Material & $\begin{array}{l}\rho \\
\left(\mathrm{g} \mathrm{cm}^{-3}\right)\end{array}$ & $\begin{array}{l}V_{\text {at }} \\
\left(\AA^{3}\right)\end{array}$ & $\begin{array}{l}M \\
\left(\mu_{\mathrm{B}} \mathrm{at}^{-1}\right)\end{array}$ & $\begin{array}{l}M^{(293 \mathrm{~K})} \\
\left(\mathrm{A} \mathrm{m} 10^{-6}\right)\end{array}$ & $\begin{array}{l}\eta_{\mathrm{n}} \\
\left(\AA^{-2} 10^{6}\right)\end{array}$ & $\begin{array}{l}\eta_{\mathrm{m}}^{(293 \mathrm{~K})} \\
\left(\AA^{-2} 10^{6}\right)\end{array}$ \\
\hline $\mathrm{Fe}$ & 7.874 & 11.8 & 2.217 & 1.713 & 8.02 & 9.95 \\
$\mathrm{Ni}$ & 8.908 & 10.9 & 0.616 & 0.490 & 9.41 & 2.73 \\
$\mathrm{Cu}$ & 8.96 & 11.8 & 0 & 0 & 6.55 & 0 \\
Precipitate & 8.96 & 11.6 & - & 0.883 & 9.04 & 5.26 \\
Matrix & 8.96 & 11.6 & - & - & 6.87 & - \\
$\Delta \eta$ & - & - & - & - & 2.17 & 5.26 \\
$\Delta \eta_{\text {m,exp }}$ & - & - & - & - & - & 4.56 \\
\hline
\end{tabular}

\subsection{SANS experiments}

As the sample was strongly textured owing to the rolling procedure during sample preparation, it was mounted with the rolling direction pointing upwards, i.e. vertical. Data were taken with a horizontal magnetic field perpendicular to the incoming neutron beam at field strengths between 72 and $520 \mathrm{mT}$. The pure spin-flip $I^{ \pm \mp}(Q)$ and non-spin-flip $I^{ \pm \pm}(Q)$ scattering intensities were extracted from the experimental intensities $I_{\text {exp }}$ according to the procedure described in $\$ 3.3$. Owing to the relatively large volume fraction of precipitates of about $22 \%$, the scattering data showed a pronounced pattern that could be recorded in relatively short time of only a few minutes.

The corrected data for the different scattering channels measured at a magnetic field of $520 \mathrm{mT}$ are shown in Fig. 5 (bottom row). The intensities are shown in linear scale. At $520 \mathrm{mT}$ the sample is practically in saturation and the scattering patterns can be well understood in a simple model of ferromagnetic single domain particles with a magnetization parallel to the field, which are arranged in a cubic paracrystalline lattice, as will be shown in the following.

The different scattering patterns and especially the change of the azimuthal anisotropy can be easily understood. They have two causes: the first is simply the spin-dependent neutron scattering cross section of magnetic nanostructures and the second is the texture of the sample itself. Even for the case of homogeneously magnetized spherical precipitates in a nonmagnetic matrix, the scattering pattern is not spherically symmetric for a partly magnetized sample because of the angular dependence of the dipole-dipole interaction between the magnetic moments of the neutron and the nanosized magnetic precipitates. Only for a fully demagnetized sample does the magnetic scattering become isotropic without any azimuthal dependency. How the scattering pattern depends on the incident and scattered spin state is amply covered in the literature (Moon et al., 1969; Price \& Sköld, 1986; Wagner \& Kohlbrecher, 2005; Wiedenmann, 2005; Honecker et al., 2010; Wiedenmann et al., 2011; Krycka, Borchers et al., 2012; Michels 
et al., 2012). Here we briefly repeat the case of homogeneously magnetized spherical particles fully magnetized parallel to the applied magnetic field, which we use to model our sample. This already explains several features of the scattering pattern. For a fully saturated sample the magnetization is parallel to the field, which was applied horizontal and perpendicular to the incoming neutron beam, i.e. $\mathbf{M}=M \mathbf{e}_{x}=(M, 0,0)$. $\mathbf{e}_{y}=(0,1,0)$ is assigned to the vertical direction and the incoming neutron beam direction $\mathbf{n}$ is assumed to be $-\mathbf{e}_{z}=(0,0,-1)$. For small scattering angles the scattering vector is practically in the plane of the detector and can be written as $\mathbf{Q}=Q(\cos \psi, \sin \psi, 0)$, where $\psi$ is the angle between the polarization direction and the scattering vector. For the magnetic scattering the so-called Halpern-Johnson vector is defined as

$$
\begin{aligned}
\mathbf{m}_{\perp} & =\gamma r_{\mathrm{e}}|\Delta \mathbf{M}| \frac{\mathbf{Q} \times(\Delta \mathbf{M} /|\Delta \mathbf{M}| \times \mathbf{Q})}{Q^{2}} \\
& =\Delta \eta_{\mathrm{m}}\left(\begin{array}{c}
\sin ^{2} \psi \\
-\cos \psi \sin \psi \\
0
\end{array}\right),
\end{aligned}
$$

with the magnetic contrast $\Delta \eta_{\mathrm{m}}=\gamma r_{\mathrm{e}}|\Delta \mathbf{M}|$. An atom with a magnetic moment of one Bohr magneton $\mu_{\mathrm{B}}$ has a scattering length of $\gamma r_{\mathrm{e}}$, where $\gamma=-1.913$ is the ratio between the neutron magnetic moment and the nuclear magnetic moment and $r_{\mathrm{e}}=2.818 \mathrm{fm}$ is the classical electron radius. Using this notation the difference in magnetization between precipitate

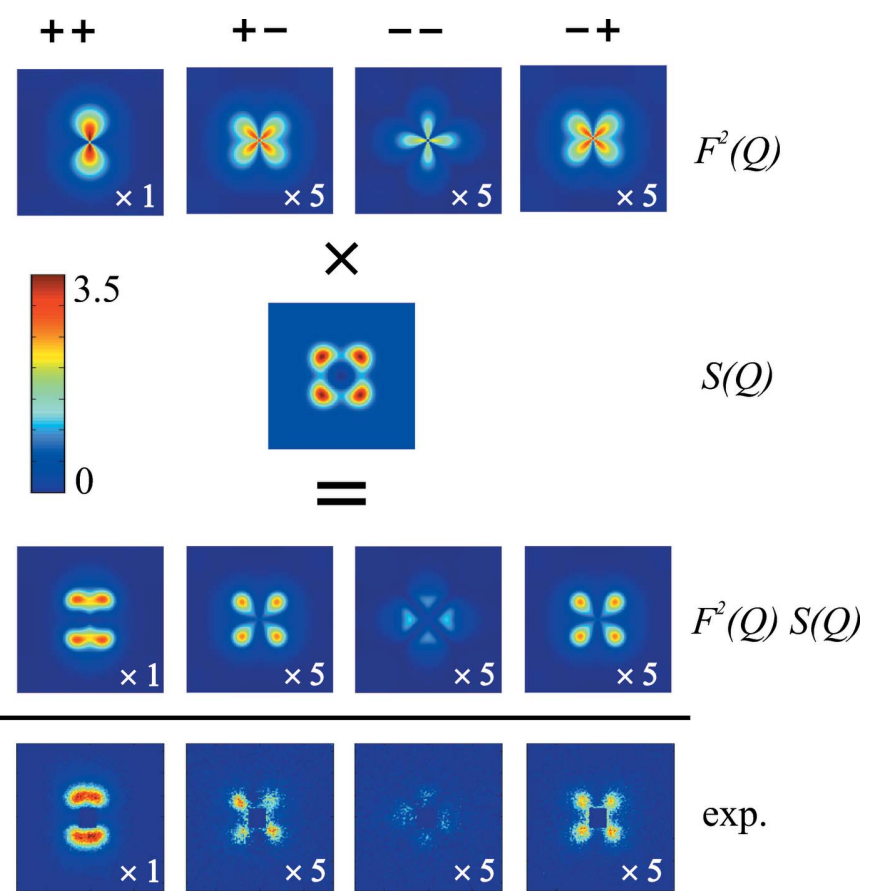

Figure 5

Comparison between model calculations and the experimental SANS data for the four scattering channels taken at a saturating magnetic field of $520 \mathrm{mT}$. Top row: scattering intensities resulting from magnetic and nuclear interaction [equations (14) and (15)]; second row: structure factor $S(Q)$; third row: product of form factor and structure factor; bottom row: experimental data. As the $I^{++}$intensity is much higher the others were multiplied by a factor of 5 before color coding. and matrix $\Delta \mathbf{M}$ has to be given in units of Bohr magnetons per volume (Squires, 2012; Price \& Fernandez-Alonso, 2013).

The four spin-dependent scattering amplitudes $F_{ \pm \pm}(\mathbf{Q}, R)$ are given by

$$
\begin{gathered}
F_{ \pm \pm}(\mathbf{Q}, R)=\left(\Delta \eta_{\mathrm{n}} \mp m_{\perp, x}\right) V_{\mathrm{p}} f(Q, R), \\
F_{ \pm \mp}(\mathbf{Q}, R)=-\left(m_{\perp, z} \mp l m_{\perp, y}\right) V_{\mathrm{p}} f(Q, R),
\end{gathered}
$$

where $\Delta \eta_{\mathrm{n}}$ is the nuclear scattering density contrast, $V_{\mathrm{p}}=\frac{4}{3} \pi R^{3}$ is the volume of the spherical particle and $f(Q, R)=3(\sin Q R-Q R \cos Q R) /(Q R)^{3}$ is its form factor. In the case of fully aligned magnetic moments in the direction of the polarization, this leads to the scattering amplitudes

$$
\begin{gathered}
F_{ \pm \pm}(Q, R, \psi)=\left(\Delta \eta_{\mathrm{n}} \mp \Delta \eta_{\mathrm{m}} \sin ^{2} \psi\right) V_{\mathrm{p}} f(Q, R), \\
F_{ \pm \mp}(Q, R, \psi)=\mp l \Delta \eta_{\mathrm{m}} \cos \psi \sin \psi V_{\mathrm{p}} f(Q, R)
\end{gathered}
$$

and the corresponding scattering intensities

$$
\begin{gathered}
I^{ \pm \pm}(Q, R, \psi)=\left(\Delta \eta_{\mathrm{n}} \mp \Delta \eta_{\mathrm{m}} \sin ^{2} \psi\right)^{2} V_{\mathrm{p}}^{2} f^{2}(Q, R), \\
I^{ \pm \mp}(Q, R, \psi)=\left(\Delta \eta_{\mathrm{m}}\right)^{2} \cos ^{2} \psi \sin ^{2} \psi V_{\mathrm{p}}^{2} f^{2}(Q, R) .
\end{gathered}
$$

The spin-dependent scattering intensities of a single spherical particle of $10 \mathrm{~nm}$ radius with a magnetization exactly parallel to the magnetic field are shown in the first row of Fig. 5.

The second contribution to the anisotropy in the scattering pattern is caused by the texture in the sample. The precipitates are arranged in a simple cubic lattice and different domains are strongly textured owing to the rolling procedure during the sample preparation (Lyon \& Simons, 1992, 1994; Wagner \& Kohlbrecher, 2005). The specifically textured simple cubic arrangement of the precipitates causes a very pronounced anisotropic structure factor $S(\mathbf{Q})$. A complete mathematical description of the structure factor of ordered nano- and mesoscale materials is given by Förster et al. (2011). The $S(\mathbf{Q})$ resulting from a fit using this model is shown in the second row of Fig. 5. The peak positions correspond to a distance between the precipitates of about $24 \mathrm{~nm}$. Assuming an average size of $10 \mathrm{~nm}$ radius one can estimate their volume fraction to about $26 \%$, which corresponds well to the equilibrium volume fraction of $22 \%$ given above.

In principle such a procedure is delicate as one cannot separate the form factor and structure factor from a single measurement without making assumptions about both of them. To experimentally separate the two contributions one has to bring disorder into the system without changing the form factor of the precipitates. For liquid samples this is often easily achieved by simple dilution, a method which obviously does not work for precipitates in alloys. In the case of magnetic nanostructures it might be possible to obtain a fully randomly oriented magnetization of the precipitates in a demagnetized sample. The random orientation of the magnetization in the precipitates can strongly suppress the contribution of the structure factor (Kohlbrecher \& Wiedenmann, 1998). We have performed measurements at low fields down to $72 \mathrm{mT}$ and observed changes in the structure factor; 
however, a model to describe these data quantitatively has not yet been worked out. It would need to include the dipoledipole interaction between the precipitates, which becomes significant at this field strength, as we discuss below. It is very likely that the dipolar interaction leads to correlations in the orientation of the magnetic moments of neighboring precipitates, even down to zero field.

In the case of monodisperse and magnetically saturated spherical particles the intensity for a dense arrangement of particles is simply the product of the squared form factor and the structure factor. The resulting scattering patterns are shown in row three of Fig. 5 and agree very well with the experimental data shown in the bottom row. For a saturated sample, the ratio between the magnetic and nuclear scattering contrast can be obtained without a detailed analysis of the scattering patterns. One simply needs to compare the total counts on the detector measured for the different incident and outgoing neutron spin directions as all contrast terms appear separately as a pre-factor in equations (14) and (15). We find a ratio between magnetic and nuclear scattering contrast of close to 2.1, which corresponds to a magnetization of $0.68 \times 10^{6} \mathrm{~A} \mathrm{~m}^{-1}$. This ratio is only slightly smaller than the one estimated from the nominal composition of precipitates and matrix of 2.4 (see Table 1), which corresponds to a magnetization of $0.883 \times 10^{6} \mathrm{~A} \mathrm{~m}^{-1}$. The slight difference might be explained by the residual $\mathrm{Cu}$ in the precipitates, which reduces the magnetization more than expected, or by the fact that demagnetization effects due to the relatively large number of atoms at the surface become relevant.

With the known absolute values for the magnetization of the precipitates, their size and their distance we can now estimate the relevant energies in our system, i.e. the dipoledipole energy between neighboring particles, their potential energy in the external applied field and their thermal energy $k_{\mathrm{BT}}$. We find that the potential energy of the magnetic moments in the applied field of $520 \mathrm{mT}$ is about 360 times larger than $k_{\mathrm{BT}}$ and about 12 times larger than the dipolar energy between neighboring precipitates. For the high-field case the assumption of a saturated sample is thus well justified. However, for the low-field measurements at $72 \mathrm{mT}$, the dipolar energy will be around half the potential energy in the external field and needs to be included in a quantitative model. As our apparatus is perfectly suited to operate at even lower fields, this opens the perspective to study dipole-dipole induced correlations by means of SANS with polarization analysis, which is however beyond the scope of this paper and will be the subject of further studies.

\section{Conclusions}

The previous section well illustrates the power of a polarization analysis of SANS of magnetic materials. A reversal of the spin state of the incoming neutrons essentially changes the proportions of the magnetic and nuclear scattering contrast of the sample under investigation [equation (14)]. But, only the polarization analysis of the scattered neutrons gives direct access to the magnetic interaction [equation (15)] and thus yields precise information on the magnetic microstructure of the sample. To perform this polarization analysis we have developed a novel compact spin filter apparatus based on protons polarized via photoexcited triplet states. It is not sensitive to stray fields and is operated at a short distance of about $30 \mathrm{~mm}$ from the sample under investigation in a common magnetic field. The setup yields an accessible $Q$ range of above $0.4 \mathrm{~nm}^{-1}$ at $0.6 \mathrm{~nm}$, even with a relatively small filter cross section, and furthermore makes guide fields between sample and analyzer superfluous. Once polarized, the filter can be operated at practically any field, giving complete freedom in the choice of field strength applied to the sample, even down to below $0.01 \mathrm{~T}$. A low field limit might be given by the filter polarization decay tolerable in a specific experiment. The following relaxation times $T_{1}$ have been measured during a recent spin filter experiment: $41 \mathrm{~h}$ at $4 \mathrm{mT}, 73 \mathrm{~h}$ at $10 \mathrm{mT}$ and $600 \mathrm{~h}$ at $550 \mathrm{mT}$.

The present system has been operated very reliably in several 2-4 week-long experiments on neutron beams and has typically achieved a flipping ratio of above 4 at an overall transmission of above $30 \%$. To further improve its performance and expand its possibilities, especially with a view to conducting spin analysis in SANS, the following development steps are considered:

(1) Having a means to quickly and efficiently reverse the filter polarization would be of clear advantage and would save the time presently needed to repolarize the filter along the opposite spin direction via the DNP process. The method of choice is the adiabatic fast passage (AFP) polarization reversal scheme, which is well known in NMR and is also applied for ${ }^{3} \mathrm{He}$ spin filters (McKetterick et al., 2011). High efficiencies should be achievable as the limiting relaxation time in the rotating frame will be very long owing to the absence of paramagnetic states in the sample, i.e. the triplets are gone once the excitation light is shut off. The AFP scheme is presently implemented.

(2) The present filter size of $\sim 5 \times 5 \times 5 \mathrm{~mm}$ is limited by the dimensions of the ESR resonant structure used. In principle much larger high-quality crystals can be grown and homogeneously excited with laser light. The intensity of the laser excitation light is far below the saturation level so that the density of created triplet states will exponentially decrease on a length scale directly comparable to the weak field absorption length, which ranges between 10 and $50 \mathrm{~mm}$ depending on the crystals' pentacene concentration. Taking into account the presently available laser power, we consider an increase of the filter size to a cross section of $10 \times 10 \mathrm{~mm}$ with $7 \mathrm{~mm}$ thickness as realistic. The ESR resonant structure then needs to be enlarged, which requires the system to be operated at a lower microwave frequency, preferably at Cband $(4-5 \mathrm{GHz})$; this has been demonstrated to be feasible (Sakaguchi et al., 2011). The increased filter cross section would yield a considerable $Q$ range for a SANS experiment. Assuming a sample with a typical size of $4 \times 4 \mathrm{~mm}$ at $32 \mathrm{~mm}$ distance from the analyzer, such a spin filter can cover a maximum scattering angle of $\pm 5.7^{\circ}$, which corresponds to a maximum accessible $Q$ of $1.04 \mathrm{~nm}^{-1}$ at $0.6 \mathrm{~nm}$ wavelength. 
With a modification of the lower part of the cryostat the distance between sample and filter could be reduced to $20 \mathrm{~mm}$, which would further increase the acceptance. These numbers compare favorably to the limits $\left(Q \simeq 1 \mathrm{~nm}^{-1}\right)$ of the most advanced ${ }^{3} \mathrm{He}$ concepts (Chen et al., 2014).

The presented spin filter based on protons polarized via photoexcited triplet states is well suited to performing polarization analysis in SANS. Placing the currently still small filter close to the sample under investigation, it can still be large enough to subtend the angular range required for a SANS study. This approach is flexible and can be significantly expanded by implementing the development steps discussed above.

\section{Acknowledgements}

We especially acknowledge technical support by Paul Schurter, whose outstanding skills have made these experiments possible. This work was supported by the Swiss National Science Foundation grant 200021_143297.

\section{References}

Abragam, A. \& Goldman, M. (1978). Rep. Progr. Phys. 41, 395-467. Aswal, V. K., van den Brandt, B., Hautle, P., Kohlbrecher, J., Konter, J. A., Michels, A., Piegsa, F. M., Stahn, J., Van Petegem, S. \& Zimmer, O. (2008). Nucl. Instrum. Methods Phys. Res. Sect. A, 586, 86-89.

Chen, W. C., Erwin, R., McIver, J. W. III, Watson, S., Fu, C. B., Gentile, T. R., Borchers, J. A., Lynn, J. W. \& Jones, G. L. (2009). Physica B, 404, 2663-2666.

Chen, W. C., Gentile, T. R., Erwin, R., Watson, S., Ye, Q., Krycka, K. L. \& Maranville, B. B. (2014). J. Phys. Conf. Ser. 528, 012014.

Crangle, J. \& Goodman, G. M. (1971). Proc. R. Soc. London Ser. A, 321, 477-491.

Delannay, L., Mishin, O. V., Jensen, D. J. \& Van Houtte, P. (2001). Acta Mater. 49, 2441-2451.

Dianoux, A.-J. \& Lander, G. (2003). Editors. ILL Neutron Data Booklet. Philadelphia: OCP Science Imprint.

Dufour, C., Fitzsimmons, M. R., Borchers, J. A., Laver, M., Krycka, K. L., Dumesnil, K., Watson, S. M., Chen, W. C., Won, J. \& Singh, S. (2011). Phys. Rev. B, 84, 064420.

Eichhorn, T. R., van den Brandt, B., Hautle, P., Henstra, A. \& Wenckebach, W. Th. (2013). Mol. Phys. 112, 1773-1782.

Eichhorn, T. R., Haag, M., van den Brandt, B., Hautle, P., Wenckebach, W. Th., Jannin, S., van der Klink, J. J. \& Comment, A. (2013). J. Magn. Resonance, 234, 58-66.

Eichhorn, T. R., Niketic, N., van den Brandt, B., Filges, U., Panzner, T., Rantsiou, E., Wenckebach, W. Th. \& Hautle, P. (2014). Nucl. Instrum. Methods Phys. Res. Sect. A, 754, 10-14.

Fitzsimmons, M. R. \& Schuller, I. K. (2014). J. Magn. Magn. Mater. 350, 199-208.

Förster, S., Fischer, S., Zielske, K., Schellbach, C., Sztucki, M., Lindner, P. \& Perlich, J. (2011). Adv. Colloid Interface Sci. 163, 5383.

Gentile, T. R., Babcock, E., Borchers, J. A., Chen, W. C., Hussey, D., Jones, G. L., Lee, W. T., Majkzrak, C. F., O’Donovan, K. V., Snow, W. M., Tong, X., te Velthuis, S. G. E., Walker, T. G. \& Yan, H. (2005). Physica B, 356, 96-102.

Glättli, H. \& Goldman, M. (1987). Neutron Scattering, Methods in Experimental Physics, Vol. 23, Part C, edited by Kurt Skøld \& David L. Price, pp. 241-286. Orlando: Academic Press.

Goossens, D. J. \& Cussen, L. D. (1999). Physica B, 267-268, 348-351.
Henstra, A., Lin, T. S., Schmidt, J. \& Wenckebach, W. Th. (1990). Chem. Phys. Lett. 165, 6-10.

Henstra, A. \& Wenckebach, W. Th. (2013). Mol. Phys. 112, 17611772.

Honecker, D., Ferdinand, A., Döbrich, F., Dewhurst, C. D., Wiedenmann, A., Gómez-Polo, C., Suzuki, K. \& Michels, A. (2010). Euro. Phys. J. B, 76, 209-213.

Kohlbrecher, J. \& Wiedenmann, A. (1998). Physica B, 241-243, 591593.

Krycka, K. L., Booth, R. A., Hogg, C. R., Ijiri, Y., Borchers, J. A., Chen, W. C., Watson, S. M., Laver, M., Gentile, T. R., Dedon, L. R., Harris, S., Rhyne, J. J. \& Majetich, S. A. (2010). Phys. Rev. Lett. 104, 207203.

Krycka, K., Borchers, J., Ijiri, Y., Booth, R. \& Majetich, S. (2012). J. Appl. Cryst. 45, 554-565.

Krycka, K., Chen, W., Borchers, J., Maranville, B. \& Watson, S. (2012). J. Appl. Cryst. 45, 546-553.

Laver, M., Mudivarthi, C., Cullen, J. R., Flatau, A. B., Chen, W. C., Watson, S. M. \& Wuttig, M. (2010). Phys. Rev. Lett. 105, 027202.

Lushchikov, V. I., Taran, Yu. V. \& Shapiro, F. L. (1969). Sov. J. Nucl. Phys. 10, 669-677.

Lyon, O. \& Simons, J. P. (1992). J. Phys. Condens. Matter, 4, 60736086.

Lyon, O. \& Simons, J. P. (1994). J. Phys. Condens. Matter, 6, 16271636.

McKetterick, T. J., Boag, S., Stewart, J. R., Frost, C. D., Skoda, M. W. A., Parnell, S. R. \& Babcock, E. (2011). Physica B, 406, 24362438.

Michels, A. (2014). J. Phys. Condens. Matter, 26, 383201.

Michels, A., Honecker, D., Döbrich, F., Dewhurst, C. D., Suzuki, K. \& Heinemann, A. (2012). Phys. Rev. B, 85, 184417.

Moon, R. M., Riste, T. \& Koehler, W. C. (1969). Phys. Rev. 181, 920 931.

Petoukhov, A. K., Guillard, V., Andersen, K. H., Bourgeat-Lami, E., Chung, R., Humblot, H., Jullien, D., Lelievre-Berna, E., Soldner, T., Tasset, F. \& Thomas, M. (2006). Nucl. Instrum. Methods Phys. Res. Sect. $A, \mathbf{5 6 0}, 480-484$.

Piller, J., Wagner, W., Wollenberger, H. \& Mertens, P. (1984). Decomposition of Alloys: the Early Stages, edited by P. Ashby, G. Haasenv \& F. Wagnerm. pp. 156-164. Oxford: Pergamon Press.

Price, D. L. \& Fernandez-Alonso, F. (2013). Editors. Neutron Scattering - Fundamentals, Experimental Methods in the Physical Sciences, Vol. 44, pp. 1-136. Amsterdam: Academic Press.

Price, D. L. \& Sköld, K. (1986). Editors. Neutron Scattering, Methods of Experimental Physics, Vol. 23. Orlando: Academic Press.

Ramazanoglu, M., Laver, M., Ratcliff, W., Watson, S. M., Chen, W. C., Jackson, A., Kothapalli, K., Lee, S., Cheong, S. W. \& Kiryukhin, V. (2011). Phys. Rev. Lett. 107, 207206.

Sakaguchi, S. et al. (2011). Phys. Rev. C, 84, 024604.

Squires, G. L. (2012). Introduction to the Theory of Thermal Neutron Scattering, 3rd ed. Cambridge University Press.

Ueland, B. G., Lynn, J. W., Laver, M., Choi, Y. J. \& Cheong, S. W. (2010). Phys. Rev. Lett. 104, 147204.

Van Strien, A. J. \& Schmidt, J. (1980). Chem. Phys. Lett. 70, 513517.

Wagner, W. (1988). Mater. Sci. Forum, 27-28, 413-420.

Wagner, W. \& Kohlbrecher, J. (2005). Modern Techniques for Characterizing Magnetic Materials, edited by Y. Zhu, pp. 65-103. Boston: Kluwer.

Wahi, R. P. \& Stajer, J. (1984). Decomposition of Alloys: the Early Stages, edited by P. Ashby, G. Haasenv \& F. Wagnerm. pp. 165-169. Oxford: Pergamon Press.

Wiedenmann, A. (2005). Physica B, 356, 246-253.

Wiedenmann, A., Gähler, R., Dewhurst, C. D., Keiderling, U., Prévost, S. \& Kohlbrecher, J. (2011). Phys. Rev. B, 84, 214303.

Wildes, A. R. (2006). Neutron News, 17(2), 17-25.

Williams, W. G. (1988). Polarized Neutrons. Oxford University Press. 